Submitted to the Human Factors and Ergonomics Society $46^{\text {th }}$ Annual Meeting

\title{
Incorporating Procedural Information in a Paired Approach Task
}

\author{
Steven J. Landry and Amy R. Pritchett
}

\begin{abstract}
This study focused on pilot performance at simultaneously flying simulated instrument approaches and self-separating from an aircraft on approach to a closely spaced parallel runway. The participant pilots flew approaches on Georgia Tech's Reconfigurable Flight Simulator and were instructed to remain within the "safe zone" - an area in which the subject aircraft was safe from collision danger and wake vortices from the other aircraft - as depicted on their navigation display. Two underlying bases for calculating the safe zone were used: a "predicted" safe zone utilizing procedural information, and an "actual" safe zone utilizing real-time information. No display effects were found for pilot performance or behavior, despite the very different implications of the two safe zone depictions. Also, pilots monitored the other aircraft closely for compliance, but could not recognize the implications of noncompliance for their own approach. This suggests that pilots have a particular, persistent, and perhaps poor, strategy for this task.
\end{abstract}

\section{Introduction}

Procedures not only represent soft constraints that regulate the environment and order behavior, but also provide information about the normative state of the environment. Pilots can use this information to make judgments and guide action. In this paper these roles of procedures are investigated by examining pilot compliance during a closely spaced parallel approach procedure, their reaction to another aircraft's noncompliance, and their use of displays that utilize procedural information.

The proposed approach procedure, "paired approaches", places two aircraft on instrument approaches to closely spaced parallel runways, with one aircraft initially offset behind the other. The trail aircraft maintains a position relative to the lead aircraft (Stone, 1996; Pritchett, 1999; Hammer, 1999). Such a position (called the "safe zone") guarantees that neither aircraft will be in danger of loss of separation within a certain time window should the other depart its approach path, and that neither aircraft will be affected by the other's wake.

Two different underlying bases can be used to determine the safe zone (Pritchett and Landry, 2001). The first uses procedural information; i.e. the "predicted" safe zone is calculated assuming that the aircraft are following a pre-specified approach procedure, thereby presenting a spatial boundary which is predictable, small and unchanging throughout the approach, but which does not account for aircraft behavior outside the approach procedure. The second is based on real-time information; i.e. the "actual" safe zone is recalculated throughout the approach based on the current states of both aircraft, thereby presenting a spatial boundary which is as large as possible for the immediate context, and constantly (sometimes rapidly) changing in size and location.

As such, this study examined the relative merits of the different underlying conceptual bases of the safe zone, which may imply different control strategies and foster different types of monitoring for unusual situations. In addition, pilot conformance and reaction to the other aircraft's nonconformance was investigated. 


\section{Method}

\section{$\underline{\text { Apparatus }}$}

Participating pilots (12 male airline pilots current or previously qualified in glass cockpit aircraft) were asked to fly approaches using Georgia Tech's Reconfigurable Flight Simulator (RFS) (Ippolito and Pritchett, 2000). The RFS is a medium fidelity simulator running on a Pentium III desktop computer. The simulator was configured with a dynamic model and cockpit systems representing a Boeing 747-400. The navigation display (ND) included an overlay of traffic information about the aircraft on the other approach and the safe zone presentations, which were displayed as staple shaped brackets (Figure 1).

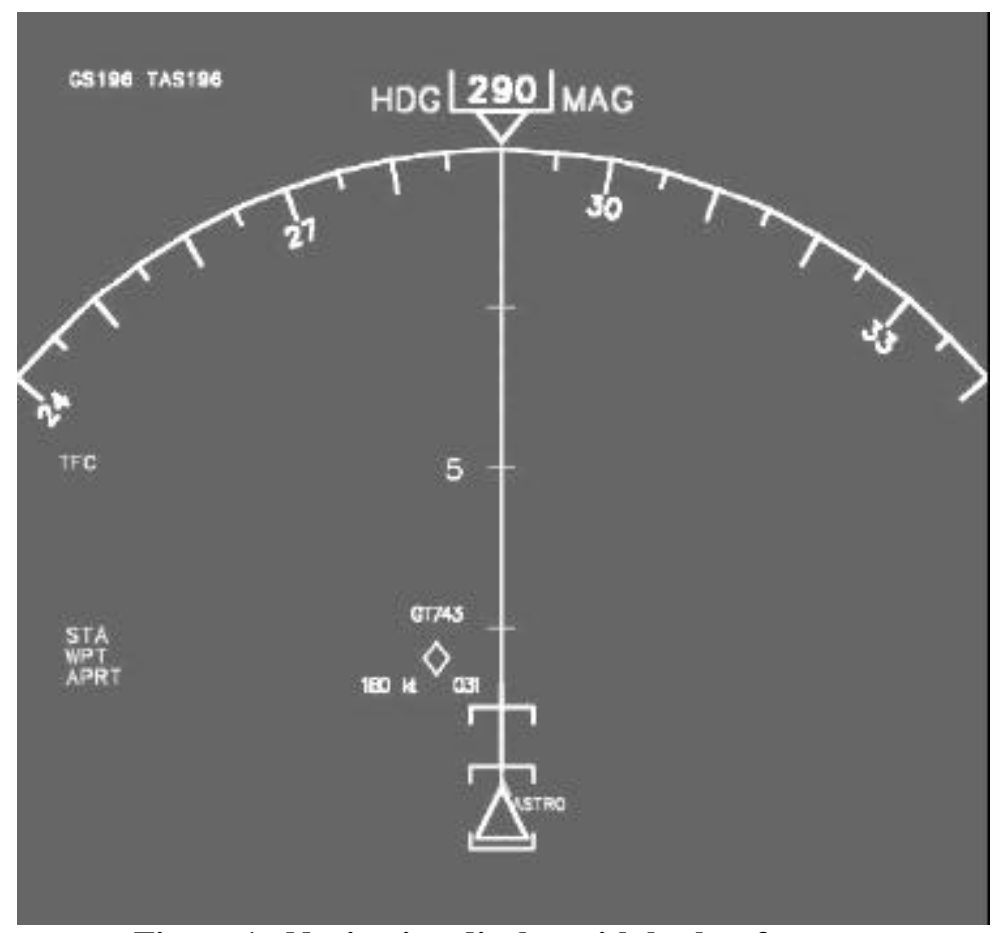

Figure 1. Navigation display with both safe zones

Procedure

Pilots were given detailed briefings on the simulator and the procedure, and given an opportunity to practice with each until they felt comfortable. In their briefing on the safe zone, it was stressed that a position within the actual safe zone was safe for the next 30 seconds from collision and wake turbulence regardless of the actions of either aircraft, while a position within the predicted safe zone was similarly safe, but only if the two aircraft were not deviating from the approach procedure. If a safe zone limit was exceeded, this protection was no longer guaranteed, and it was recommended that a missed approach be executed in this case. The missed approach procedures were provided on the approach plate, and indicated both a climb and a turn away from the other approach path.

The participants were instructed to fly an instrument landing system approach, while remaining within the safe zone. The participant pilots flew the trail aircraft, with the lead aircraft being a scripted pseudo-aircraft. Each run began at approximately 20 miles from runway threshold on the localizer and at approximately 200 knots true air speed (KTAS). The participants were instructed that ATC had told them (and the lead aircraft) to maintain 180 KTAS, plus or minus 10 knots, until 5 miles from runway threshold, where they could slow to their normal approach speed of 148 KTAS. 


\section{Experiment Design and Independent Factors}

Each participant pilot flew 10 data collection runs. The first nine runs represented a twofactor design with three safe zone displays and three blunder types. The three displays refer to the conceptual basis of the safe zone, as follows:

- Predicted safe zone display: The predicted safe zone was shown on the ND.

- Actual safe zone display: The actual safe zone was shown on the ND.

- Both safe zones display: Both safe zones were shown on the ND, allowing the pilot to directly compare the two types of information. In this case the pilots were briefed that they could exceed the predicted safe zone limits as long as they remained within the actual safe zone limits.

The blunder type refers to the type of noncompliance committed by the lead aircraft:

- No noncompliance: a baseline in which the lead aircraft complied with all procedural restrictions.

- Speed noncompliance: The lead aircraft slowed substantially below the approach procedure's minimum allowed speed, as if this aircraft were configuring and attaining final approach speed 5-10 miles before allowed by approach procedures.

- Lateral noncompliance: The lead aircraft turned toward and crossed the participant's approach path, in the form of a turn to a new heading commonly used as a blunder model.

Once the participant completed these nine runs, he flew a tenth run with one of the three safe zone displays in a combined deviation scenario: specifically, the lead aircraft first slowed below the minimum allowed procedural speed, and then the lead aircraft also turned toward and crossed the trail aircraft's approach path.

In addition to observations of pilot behavior and comments, the following quantitative measurements were taken and will be discussed in this paper:

- Pilot detection of non-procedural actions by the lead aircraft, and the ability of the pilot to describe what the lead aircraft did; and

- Approach stability as indicated by the variation about mean in the throttle movements, elevator movements, and glideslope tracking

\section{Results and discussion}

There were no significant main effects for detection of noncompliance or for any control behavior. This implies that pilots' performance at detecting the lead noncompliance and at maintaining stability on approach did not vary with different safe zone presentations.

There are several consequences of this finding. First, since the predicted safe zone is not accurate when the lead aircraft is violating the approach procedure (such as departing its approach course), a logical strategy for the pilots when given only the predicted safe zone display would be to monitor for noncompliance and initiate a go-around should it be detected. Conversely, since the actual safe zone does not depend upon the lead aircraft's compliance to procedure, a logical strategy would be to track the safe zone and not be concerned with the other aircraft's compliance. However, the lack of significant results for detection of blunders indicates that they were probably not using these strategies.

It appeared instead that pilots were monitoring for compliance regardless of display type. All twelve pilots mentioned and made frequent use of the text indication of speed on the navigation display, and four pilots indicated that a lateral trend indicator would be useful. These observations suggest that the pilots were concerned about compliance, but this concern was independent of the display of the safe zone. It seems that the pilots were worried about the overall safety of the approach ("Am I closing on the lead aircraft? Is the lead aircraft turning towards me?") rather than about the effect of compliance on the safe zone. 
More generally, the pilots' use of the displayed position of the safe zone appeared to be independent of the conceptual basis for the display. Eight pilots waited until after leaving the safe zone before executing a missed approach, despite it being inevitable in cases where their speed exceeded the lead's speed. Three other pilots began the missed approach procedure once it was inevitable that they would leave the safe zone, and the remaining pilot initiated missed approach once he perceived that the lead aircraft was not complying with the procedure in some way. In addition, when the lead aircraft departed its approach course, many pilots chose to remain within the (now inaccurate) predicted safe zone, just as they had done when presented with just the actual safe zone (which was still accurate under these circumstances). These findings suggest that the pilots were using the display of the safe zone as a "go/no-go" indicator; if they could remain within whichever safe zone was displayed they would continue on the approach, otherwise they would go around. This lack of distinction concerning the conceptual basis for the safe zone may have been encouraged by the similar format used for their presentation, which differed only in color (predicted was green, actual was yellow).

Another logical strategy when both safe zones were displayed would be to use the predicted safe zone to stabilize position within the safe zone. As essentially a "worst-case" safe zone, remaining within the predicted safe zone would also ensure that they remained within the actual safe zone for the duration of the approach. In fact, only two pilots made specific attempts to remain behind the predicted safe zone brackets when both brackets were available, and two other pilots indicated that they felt that the predicted safe zone display was not useful. This may be because the pilots felt that they could remain behind the actual safe zone without difficulty, although the actual safe zone could change length and position rapidly when the lead aircraft maneuvered.

There were a number of additional interesting observations concerning the participant pilots' compliance to approach procedures. First, there was a significant time lag between exiting the safe zone and initiating a go around, even in cases where the subjects could predict that they would depart the safe zone. In addition, one pilot chose to remain on his approach path in several scenarios despite leaving the safe zone, as he felt it unsafe to perform a go-around. Two other pilots elected to not fly the missed approach as published when the other aircraft had crossed in front of them (and therefore was flying in the same direction as the missed approach course). Pilots seemed to be searching for additional confirming (or dissenting) information to support their decision. This observation is abundantly confirmed in a number of studies concerning compliance - pilots cannot be assumed to act automatically to procedures or alerts (Pritchett, 1999; Ockerman and Pritchett, in press). However, the uncertainty of this environment may have justified the delays and the deviations from normal go-around procedures.

Another interesting result is that pilots have a wide variety of methods of maintaining spacing for current visual closely spaced parallel approaches. Each of the twelve pilots had a different target separation, ranging from nose-tail to 5 miles. Other responses included keeping the other aircraft at a 45-degree angle and keeping whatever spacing ATC had provided. Four pilots mentioned keeping vertical separation from the other aircraft. These strategies are problematic, as, for example, maintaining nose-tail separation is demonstrably unsafe - in this position a sudden turn by the lead aircraft can cause a loss of separation within ten seconds.

\section{Conclusions}

Pilots use procedural information, but they are not bound by it. The circumstances under which they will and will not comply is difficult to predict, and is idiosyncratic. An obvious consequence is that relying on compliance is inadvisable. A less obvious consequence is that pilots expect deviations from procedures, whether though error or through mitigating circumstances. They therefore want to see information that allows them to monitor for these deviations. Successful detection of noncompliance, however, does not guarantee that pilots will be able to interpret the consequences of that noncompliance. 
The monitoring that pilots used for this approach appeared to be related to a relatively simple strategy. This strategy required only basic position, speed, and trend information for the other aircraft. Pilots generally complied with the instructions concerning remaining within the safe zone, but based their judgment of safety on their own strategy, despite understanding that the safe zone represented a better model of safety.

\section{References}

Hammer, J., 1999. Case study of paired approach procedure to closely spaced parallel runways. Air Traffic Control Quarterly, Vol. 8(3), 223-252.

Ippolito, C. and Pritchett, A., 2000. Software architecture for a reconfigurable flight simulator. AIAA Modeling and Simulation Technologies Conference: Denver, CO.

Ockerman, J. and Pritchett, A. (in press). A review and reappraisal of task guidance: Aiding workers in procedure following. International Journal of Cognitive Ergonomics.

Pritchett, A. and Landry, S., 2001. Two studies of paired approaches. Presented at The 2001 Air Traffic Management Research and Design Workshop: Sante Fe, NM.

Pritchett, A., 1999. Pilot performance at collision avoidance during closely spaced parallel approaches. Air Traffic Control Quarterly, Vol. 7(1) , 47-75.

Stone, R., 1996. Paired approach concept. In Waller, M.C. and C.H. Scanlon (Eds.) Proceedings of the NASA Workshop on Flight Deck Centered Parallel Runway Approaches in Instrument Meteorological Conditions: NASA Langley Research Center. 